\title{
Guidance Document for Structured Reporting of Diuresis Renography
}

\author{
Andrew T. Taylor, MD*, M. Donald Blaufox, MD, PhD ${ }^{\dagger}$, Diego De Palma, MD‡, Eva V. \\ Dubovsky, MD, PhD§, Belkis Erbaş, MD", Anni Eskild-Jensen, MD|, Jørgen Frøkiær, MD, \\ DMSci $^{\star *}$, Muta M. Issa, MD, MBA ${ }^{\dagger \dagger}$, Amy Piepsz, MD, PhD ${ }^{\ddagger \ddagger}$, and Alain Prigent, MD, PhD $\$ \S$ \\ "Department of Radiology School of Medicine, Atlanta, GA \\ tDepartment of Nuclear Medicine, Albert Einstein College of Medicine, New York, NY \\ ¥Nuclear Medicine Department, Circolo Hospital and Macchi Foundation, Varese, Italy \\ §Department of Radiology, University of Alabama, Birmingham, AL \\ IDepartment of Nuclear Medicine, Hacettepe University Medical School, Ankara, Turkey \\ IDepartment of Nuclear Medicine, Aarhus University Hospital, Aarhus, Denmark \\ ${ }^{* *}$ Department of Clinical Physiology and Nuclear Medicine, Aarhus University Hospital—Skejby, \\ Aarhus N, Denmark \\ ttDepartment of Urology, Emory University School of Medicine, Urology Services, Veterans \\ Administration Medical Center, Atlanta, GA \\ ¥¥Department of Radioisotopes, University Hospital, St-Pierre, Brussels, Belgium \\ §\&University of Paris-Sud 11, Department of Biophysics, Nuclear Medicine and Clinical \\ Neurophysiology, Bicêtre Hospital, Assistance Publique-Hôpitaux de Paris, Paris, France
}

\begin{abstract}
The International Scientific Committee of Radionuclides in Nephro-urology (ISCORN; http://www.iscorn.org) began in the late 1960s as an independent group of physicians and scientists working to facilitate radionuclide renal research and the practice of renal nuclear medicine. This group has subsequently issued a series of Consensus reports and has now developed a Guidance Document for quality assurance and structured reporting of diuresis renography in adults. ISCORN chose diuresis renography for its first Guidance Document for several reasons: suspected obstruction is the most common reason for referral, most radionuclide renal studies are conducted at institutions that perform fewer than 3 studies per week, and a large percentage of radionuclide renal studies are interpreted by physicians with limited training in nuclear medicine. An additional rationale was the observation that diuresis renography reports from ISCORN member institutions showed marked variation in the elements included in the reports as well as considerable variation in how the results were communicated to the referring physicians. Reports that omit the essential components of the procedure, that lack the necessary elements required for quality assurance and interpretation, that fail to indicate the rationale supporting the conclusions, and that fail, in some cases, to even clearly state the conclusions are a disservice to patients and reflect badly on nuclear medicine and radiology. To address these concerns, this Guidance Document was developed through an iterative series of comments and
\end{abstract}


questionnaires regarding the reporting structure and importance of specific elements in the report. Panelists were asked to categorize each element as essential, recommended but not essential, local option (possibly useful but without sufficient data to support a higher ranking), and unnecessary (does not contribute to quality assurance or scan interpretation). Each element was independently scored by panelists without access to the individual scores of the other members or knowledge of the identity of panel members making specific comments. A majority vote was required to place an element in a specific category. The Guidance Document recommends a reporting structure organized into indications, clinical history, study procedure, findings and impression. The Guidance Document also specifies the elements considered essential or recommended in each of the reporting categories and provides a brief discussion of specific elements. Few elements, however, achieved unanimous agreement and the panel recognizes the need for innovation and the possibility of constraints imposed by local circumstances. Consequently, the Guidance Document is not intended to be restrictive but rather to provide a basic structure and rationale for diuresis renography reports in adults so that a report: (1) communicates the results to the referring physician in a clear and concise manner designed to optimize patient care; (2) contains the essential elements required to evaluate and interpret the study; (3)clearly documents the technical components of the study necessary for accountability, quality assurance and reimbursement; and (4) encourages clinical research by facilitating better comparison and extrapolation of results between institutions.

The International Scientific Committee of Radionuclides in Nephro-urology(ISCORN) began in the late 1960s as an independent group of nephrologists, urologists, renal physiologists, radiochemists, radiologists, and nuclear medicine physicians dedicated to radionuclide renal research and the practice of renal nuclear medicine. ${ }^{1}$ This group has subsequently issued Consensus reports on renovascular hypertension, ${ }^{2}$ clearance measurements, ${ }^{3}$ diuresis renography, ${ }^{4}$ quality control of quantitative measurements obtained from the renogram, ${ }^{5}$ renal cortical scintigraphy in children with urinary tract infection, ${ }^{6}$ technical aspects of renal transplant scintigraphy, ${ }^{7}$ and transit time analysis ${ }^{8}$ and has now developed a Guidance Document for quality assurance and structured reporting of diuresis renography in adults.

ISCORN chose diuresis renography for its first Guidance Document because a recent review of almost 2000 renal renography studies indicated that suspected obstruction was the most common reason for renography referral (A.T. Taylor, unpublished results). An additional rationale for a diuresis renography guidance document was the fact that most radionuclide renal studies are conducted at institutions that perform fewer than 3 studies/week ${ }^{9}$ and, in the United States, are often interpreted by physicians with limited training in nuclear medicine. Finally, diuresis renography reports from panel member institutions with a strong academic interest in renal nuclear medicine showed considerable variation in the elements included in the reports as well as considerable variation in how the results were communicated to the referring physicians. Diuresis renography reports that fail to provide the details of the procedure, fail to provide the elements and rationale supporting the conclusions, and that fail, in some cases, to even clearly state the conclusions are a disservice to our patients undermine the field of renal nuclear medicine and reflect badly on nuclear medicine and radiology in general.

The Guidance Document specifies a reporting structure and identifies the elements that should be included in the report. Few elements, however, achieved unanimous agreement and the panel recognizes the need for innovation and the possibility of constraints imposed by local circumstances. The Guidance Document is not intended to be restrictive but rather to provide a basic structure and rationale for diuresis renography reports in adults so that a report: (1) communicates the results to the referring physician in a clear and concise manner 
designed to optimize patient care; (2) contains the essential elements required to evaluate and interpret the study;(3) clearly documents the technical components of the study necessary for accountability, quality assurance and reimbursement; (4) encourages clinical research by facilitating better comparison and extrapolation of results between institutions.

\section{Methods}

\section{Panel Composition}

The panel was composed of current or past members of the ISCORN scientific committee and expanded to solicit additional input from urologists.

\section{Consensus Methodology}

The methodology was a simplified version of the Delphi approach similar to that used to develop the guidelines for brain single-photon emission computed tomography and angiotensin-converting enzyme inhibition renography. ${ }^{2,10}$ After soliciting input and sample diuresis renography reports from panel members, the chair (A.T.T.) prepared an Excel spreadsheet initially organized into 3 subject headings: (1) procedure (what information should be included to describe the procedure); (2) findings (which measurements and descriptors should be included in the report); and (3) impression (what components should be stated in the impression). Each of these subject headings contained 20, 25, and 26 elements, respectively. Panelists were asked to rate each of the elements as (1) essential (must be included in the report), (2) recommended, (3) local option (possibly useful but without sufficient data to support a higher ranking), and (4) not necessary (does not contribute to quality assurance or scan interpretation). There was a fourth heading for the insertion of free text called "Comments and suggestions."

Elements were assigned to specific categories (essential, recommended, local option, not necessary) on the basis of majority vote. For example, one element in the second round was "Report MAG3/DTPA/Cr-51 EDTA/EC clearance and normal range." Three panelists voted "essential," 5 voted "recommended," one voted "local option," and one abstained. Because only 3 of 9 voting panelists thought this element was essential but 8 of 9 thought it was either essential or recommended, this element was place in the recommended category.

After the first round, results were tallied, and the scores were provided to the panel members along with a list of all comments and suggestions. With the exception of the chair, panel members were blinded to individual scores and the identity of panel members making specific comments. In addition to the first round scores and a list of comments, panelists received a second Excel sheet with the same headings containing 22, 38, and 33 elements in each heading, respectively. Elements were added that had been omitted, ambiguous elements were clarified and many of the same elements included in the first round were also submitted for a second round of scoring now that panel members had access to the comments and scores of the other panelists. The second round also contained yes/no questions regarding the structure and content of the report as well as a section for comments.

On the basis of the first 2 rounds of scoring and answers to the yes/no questions, the chair prepared and e-mailed a draft document to the panelists along with the second round scores, second round comments and a third excel spreadsheet that contained 19 elements and an additional category called "Clinical Information." Panelists returned their critiques of the draft document, their third round scores, and their comments to the chair, who prepared and presented a second draft document at the 14th International Symposium of Radionuclides in Nephrology held in Mikulov, Czech Republic, in 2010. Participants in the audience were invited to make formal or informal suggestions. The second draft document was also circulated to the panelists along with a fourth excel spreadsheet with 10 elements that 
addressed issues not fully resolved in the first 3 rounds of scoring. Panelists critiqued the second draft document and returned their suggested revisions and the fourth round of scoring to the chair. On the basis of the comments, critiques and the fourth round of scoring, the chair prepared a third draft document which was submitted to the panelists for final comments and approval.

\section{Results}

The panel voted to adopt the American Society of Nuclear Cardiology reporting structure, which organizes the report into indications, clinical history, study procedure, findings and impression. ${ }^{11,12}$ The elements that were considered essential or recommended in each of these report categories are listed below under the appropriate category accompanied, when indicated, by a short explanation.

\section{Indications}

\section{Essential}

1 Reason for referral. The reason for referral is the justification for performing the study and should indicate the clinical question the study is designed to answer.

\section{Clinical History \\ Essential}

2 Age and gender: these elements should be included in the report unless they are already contained in the patient descriptors.

3 State most recent serum creatinine measurement and date. Normal kidneys should have a good diuretic response to $40 \mathrm{mg}$ of furosemide, whereas patients with azotemia may need greater doses to achieve an adequate diuretic response. ${ }^{13-15}$ Knowledge of the patient's renal function can be important in selecting the dose of furosemide and in interpreting the drainage pattern.

$4 \quad$ List current diuretic medications. Diuretic medications increase the likelihood that the patient may be relatively depleted of volume; volume-depleted patients may have a blunted response to furosemide and may need additional hydration. Furthermore, some patients with impaired renal function may be taking furosemide on a routine basis and be less likely to have an adequate diuretic response to a standard $40 \mathrm{mg}$ dose of furosemide. ${ }^{13}$ Knowledge of a patient's diuretic medication status alerts the nuclear medicine physician to these possibilities and can be factored into the interpretation of the study.

5 Summarize relevant results of recent imaging procedures (computed tomography, ultrasound, magnetic resonance imaging, etc)used to evaluate the kidney or ureters and date of procedure. Knowledge of findings from recent imaging procedures (renal size, presence or absence of hydronephrosis, hydroureter, degree of hydronephrosis or hydroureter, stones, stone location, masses, etc) often contribute to the interpretation of the images, renogram curves and quantitative data obtained during diuresis renography.

6 Summarize any relevant urological procedures (pyeloplasty, stent placement, stent removal, percutaneous nephrostomy) and date of procedure. Knowledge of relevant urological procedures is often essential in the interpretation of the images, renogram curves and quantitative data obtained during diuresis renography. 


\section{Recommended}

7 Summarize relevant results of most recent renal scan and provide the date of the study.

8 If there is no recent creatinine available, state this in the report. This recommendation places an additional obligation on the interpreting physician to actually check the medical records to determine if a serum creatinine has been obtained when this glomerular filtration rate marker is not provided by the ordering physician. A check of the medical records may uncover a recent creatinine which may impact the interpretation of the images, curves and quantitative data or result in administration of a higher dose of furosemide. If pertinent history is not provided or available, some authors have recommended that this omission be explicitly stated in the report; a statement that pertinent history is not available reflects current medico legal advice, sends a subtle message to the ordering physician and may convey appropriate diagnostic uncertainty. ${ }^{16}$

\section{Procedure}

\section{Essential}

9 Describe any additional hydration in the department (method of administration [oral, intravenous], volume of additional hydration, and timing relative to tracer injection) or state that no additional hydration was provided. Adequate hydration is an important component of the study. Multiple consensus reports and guidelines recommend supplemental hydration for adults and children when the patient arrives in the department. $4,5,17-19$ The report should specify any additional hydration in the department (oral, intravenous, type of hydration, volume and timing relative to tracer injection). This component documents adherence to guidelines, facilitates obtaining a diagnostic study, and provides a reminder to the nuclear medicine physician to implement this component in his/ her practice to optimize study quality.

10 State radiopharmaceutical and dose administered. Previous consensus reports recommend tubular agents for diuresis renography in adults and children ${ }^{4,17,18}$; the panel reaffirmed the recommendation to use tubular tracers $\left({ }^{99 \mathrm{~m}} \mathrm{Tc}-\right.$ MAG3, ${ }^{99 \mathrm{~m} T c-E C, ~ I-123 ~ O I H) ~ i n ~ p a t i e n t s ~ w i t h ~ s u s p e c t e d ~ o b s t r u c t i o n . ~ T h e r e ~}$ commended dose range in adults for ${ }^{99 \mathrm{~m}} \mathrm{Tc}-\mathrm{MAG} 3, \mathrm{EC}$, and DTPA is $70-200$ MBq. $^{5}$

11 Describe the imaging procedure (1 or 2 stage acquisition), including the time of acquisition. Diuresis renography can be performed as a single acquisition with $40 \mathrm{mg}$ of furosemide administered intravenously before, at the beginning or during the acquisition or it can be performed as a 2 stage acquisition consisting of a baseline acquisition followed by a furosemide acquisition if the baseline acquisition does not exclude obstruction. All 3 approaches are acceptable.,19-22

12 State if furosemide was not administered. This recommendation only applies to protocols with separate baseline and furosemide acquisitions where the furosemide acquisition may not be obtained if the baseline acquisition excludes obstruction.

13 If furosemide was administered, state dose of furosemide and timing of furosemide $(\mathrm{F})$ relative to radiopharmaceutical administration $(\mathrm{F}-15, \mathrm{~F} 0, \mathrm{~F}+$ $10, \mathrm{~F}+20$, etc). The nomenclature " $\mathrm{F}-15$ " means that furosemide was administered 15 minutes before tracer administration, " $\mathrm{F} \mathrm{cm}$ " means that 
furosemide was administered simultaneously with the tracer, and the " $\mathrm{F}+$ " notation specifies the number of minutes elapsed after tracer administration before furosemide was given.

14 State that data were recorded and processed with a computer to generate the renogram curves, relative uptake, $T_{\max }$, and $T_{1 / 2}$. If other quantitative variables were generated and used to interpret the study, list these additional variables. Generation of renogram curves and calculation of relative uptake, $T_{\max }$, and $T_{1 / 2}$ are considered essential diagnostic components; their measurement should be documented in the report along with any additional variables locally considered important in interpreting the study.

15 If a clearance measurement was performed, state method of clearance measurement (camera based, single plasma sample, multiple plasma samples). Clearance measurements provide an independent measure of renal function; knowledge of renal function can be important in selecting the dose of furosemide and in interpreting the images, renogram curves and quantitative data. Changes in renal function on sequential studies can provide important diagnostic and prognostic information.

16 State if bladder catheter, urinary diversion or nephrostomy drainage is present. The presence of a bladder catheter, urinary diversion or nephrostomy tube can affect urine drainage and the interpretation of the study and must be documented. Furthermore, if a nephrostomy tube is present, it should be noted if the tube is clamped or unclamped at the time of the study.

\section{Recommended}

17 State the patient position during acquisition. Most diuresis renography studies are performed with the patient supine; however, studies can be performed with the patient in an upright, sitting or semirecumbent position. The patient's position during acquisition should be noted.

18 State background regions of interest (ROIs) and method of calculating relative uptake. The choice of the background ROI and method of background correction can affect the relative uptake; a peri-renal background slightly separated from the kidney is an acceptable approach. 5,24 Automated backgrounds, even when they can be modified by the operator, minimize intra- and inter observer variability and have the advantage of reproducibility.

19 State method of measuring $T_{1 / 2}$. The method of calculating the $T_{1 / 2}$ has not been standardized. Because the $T_{1 / 2}$ can vary depending on the parameters and method used to make the calculation (count based, linear fit, exponential fit, etc), the report should state the method used to make the measurement.

20 In addition to the $T_{1 / 2}$, state that another measure of excretion was obtained, such as a gravity assisted postvoid image, output efficiency, the residual or postvoid renal counts normalized to the 1-2 minutes renal counts (Nora) or the residual or postvoid renal counts normalized to the maximum counts. These or similar additional measures accompanied by normal values appear to provide better discrimination between obstructed, equivocal and non-obstructed kidneys in children and adults than the $\mathrm{T}^{1 / 2}$ alone. ${ }^{20,25-31}$

21 State that a postvoid kidney image was obtained. Pressure differences between the renal pelvis and bladder, normal peristalsis, and gravity facilitate drainage from the renal pelvis. Emptying the bladder maximizes the pressure difference between the renal pelvis and bladder and usually requires the patient to assume 
an upright posture thus allowing gravity to contribute to urine drainage. An empty bladder and gravity assume increased importance when there is delayed drainage from a supine patient or if there is abnormal peristalsis.

22 State that the voided volume was measured. Voided volume can be easily determined by collecting the urine volume in a calibrated container (see comment 38).

23 State that the residual urine volume was measured. The residual urine volume can be calculated from the pre- and post-void bladder counts and the voided volume (see comment 39 ). ${ }^{32}$

24 State that patient voided immediately before the study. This statement documents quality assurance. A full bladder may delay upper tract emptying and give false positive results. ${ }^{4}$ Furthermore, if the patient starts the study with a full bladder, there is a much greater likelihood that the study will be interrupted and incomplete because of the patient's need to void before the conclusion of the study.

25 State that the urine flow rate was measured. Urine flow rate can be calculated by noting the time of voiding before the study, the time of voiding at the conclusion of the study and measuring the urine volume at the conclusion of the study. Urine volume divided by the time in minutes between the 2 voidings provides the urine flow rate in $\mathrm{mL} / \mathrm{min}$ (see comment 40 ).

26 State that an image was obtained over the injection site if a clearance measurement was obtained. Dose infiltration can invalidate plasma or camera based clearance measurements. An image over the injection site is an important quality control procedure when clearance measurements are performed.

27 Include the relevant images, renogram curves and quantitative data with the reports. Panelists agreed that images, renogram curves and quantitative data could be an important component of the report but recognized that not all reporting systems allow the inclusion of imaging or graphic data.

\section{Findings}

\section{Essential}

28 Comment on the quality of the study; describe any problems.

29 State the relative uptake of the 2 kidneys rounded to the nearest percent (ie, 53\% and $47 \%$ vs $53.3 \%$ and $46.7 \%$ ). It is misleading to the referring physician to suggest that the measurement of relative renal function is accurate to the 10th of a percentage. Although some software programs may generate relative renal function as a fraction of a percentage, the values should be rounded off to the nearest whole percent.

30 Describe the relative size and shape of the kidneys.

31 Qualitatively describe the renogram curves (uptake and washout for each kidney).

32 Note and describe location of any pre-and/or postfurosemide tracer retention in the calyces, renal pelvis and/or ureters. The presence or absence of tracer retention in the calyces, renal pelvis and/or ureters pre-and/or postfurosemide represent important findings excluding or supporting the diagnosis of obstruction. 
32 Note time to peak height $\left(T_{\max }\right)$ of the whole kidney renogram curve. The $T_{\max }$ is prolonged in obstructed kidneys although the whole kidney $T_{\max }$ can also be prolonged in patients with dehydration or impaired function.

34 Note T1/2 for whole-kidney ROIs. This measurement is widely obtained, is often described in publications on diuresis renography and is recommended by the panel. It should be noted that reduced renal function will often result in a prolonged parenchymal transit time and a prolonged $T_{1 / 2}$ even in the absence of obstruction. To minimize the effect of reduced renal function on the whole kidney $T_{1 / 2}$, some investigators evaluate washout by placing a ROI around the retained tracer in the collecting system specifically excluding the parenchyma. More studies, however, are needed to evaluate the utility of this approach and its use is considered a local option.

\section{Recommended}

35 Report the Tc99m MAG3, EC, DTPA, or I-123 OIH clearance and normal range. The serum creatinine is an insensitive measure of renal function until renal function is reduced by approximately $50 \% .{ }^{33}$ The creatinine clearance as a measure of Glomerular filtration rate suffers from a lack of accuracy and poor precision. $3,33,34$

36 Describe drainage from kidneys after voiding. The rationale for a postvoid image is described in 20 and 21. Drainage is an important diagnostic criterion in excluding or diagnosing obstruction.

37 In addition to the $T_{1 / 2}$, report another quantitative measure of drainage. Supplemental drainage parameters could include the residual renal counts normalized to (divided by) the 1-2 minutes renal counts or maximal renal counts, postvoid renal counts/1-2 minutes or maximal counts, counts in the last time point of the postfurosemide acquisition/1-2 minutes counts or maximal counts, postfurosemide postvoid renal counts/1-2 minutes counts or maximal counts, or output efficiency (see 20 and 21).

38 Report the voided volume. The voided volume during the period of the study provides an index of hydration and is a necessary measurement to calculate residual urine volume and urine flow rate. If the patient has normal renal function and receives an intravenous injection of $40 \mathrm{mg}$ of furosemide, the patient should produce $200-300 \mathrm{~mL}$ of urine by the end of the $20-30$ minutes. ${ }^{35}$ To obtain an accurate measurement of the urine produced during the study period, it is essential for the patient to void immediately before the study.

39 Report the residual urine volume. A large residual urine volume may be clinically important and indicate an unrecognized problem in addition to adversely affecting the rate of tracer washout from the collecting system. For example, patients with prostatic hypertrophy may have substantial postvoid residuals and patients with diabetes may present with a diabetic cystopathy, bladder distention and an increased risk of reflux and pyelonephritis. Normal values are reported by Esteves et al. ${ }^{30}$

40 Report the urine flow rate. A urine flow rate of $1-3 \mathrm{~mL} / \mathrm{min}$ documents adequate hydration; dehydrated patients often show little improvement in radionuclide washout because of a poor diuresis. ${ }^{36}$

41 Report the presence or absence of left or right flank pain following furosemide. The appearance of diuresis induced flank pain can contribute to the diagnosis of 
obstruction. The absence of diuresis induced flank pain is a negative finding but the absence of flank pain does not exclude obstruction.

\section{Impression}

\section{Essential}

42 If there are technical problems with study, state problems.

43 State relative uptake.

44 Describe shape and size and position of the kidneys if a kidney is abnormal.

45 State the conclusion regarding the presence or absence of obstruction (obstructed, indeterminate, nonobstructed) with a brief summary of the findings supporting the conclusion. The rationale for the conclusion is even more important when the interpretation is indeterminate.

46 Word the report to indicate the level of confidence in the conclusion. Urologists may intervene or not intervene based on the report; consequently, the referring physician would like as clear and concise a statement of certainty as possible. Reports are sometimes unnecessarily qualified. If the impression is, "Left kidney obstruction," there is no need to qualify the impression by wording, such as, "Findings are present that are consistent with the diagnosis of left kidney obstruction." This type of qualified impression suggests uncertainty in the impression and is inappropriate unless uncertainty actually exists; if the interpreting physician is uncertain, the reasons for the uncertainty should be clearly expressed.

47 Provide a comparison to the previous study.

\section{Recommended}

48 State MAG3/DTPA/EC/OIH clearance and normal range.

49 State residual volume.

50 State urine flow rate.

\section{Comments}

Height and Weight-Height and weight are necessary to estimate renal depth with the use of a camera-based technique to calculate DTPA, EC, or MAG3 clearance. These values are also necessary to normalize clearance measurements to a body surface area of $1.73 \mathrm{~m}^{23}$ and should be included when clearance measurements are provided.

Renal Perfusion-Levels of activity up to $370 \mathrm{MBq}$ of MAG3, EC, or DTPA are sometimes administered to obtain enough counts for 1- to 3-second images of the bolus as it transits the aorta and renal arteries and to calculate renal flow indexes. Renal perfusion based on 1- to 3-second images of the bolus as it transits the aorta and renal arteries has not been demonstrated to provide useful information for interpreting diuresis renography studies and most panelists agreed that cluttering the report with relative perfusion data or a perfusion description based on the 1- to 3-second images is unnecessary. This conclusion mirrors the consensus report on quality control of quantitative measurements which states that, "renal blood flow quantitation requires higher injected activity without adding any useful clinical data, except for the kidney transplant."

Flank Pain-If unilateral or bilateral flank pain occurs after the administration of furosemide, the panelists recommend its inclusion in the findings of the report. If flank pain 
is not mentioned, the referring clinician can infer that flank pain was not present. This inference is probably a reasonable one, but it assumes that the patient was questioned regarding flank pain and that no flank pain occurred. Reporting the absence of flank pain provides greater assurance that the presence or absence of flank pain following furosemide was noted. Panelists were evenly divided regarding the need to report the absence of flank pain with 5 recommending inclusion in the report and 5 indicating that this statement was not necessary.

Diuresis Renography in Infants-The primary focus of this guidance document is quality assurance and structured reporting in adults. Infants present special issues, particularly related to antenatal hydronephrosis and a separate guidance document for infants and children is under development.

Radiation Risk-Some reports include a statement regarding radiation and radiation risk. When the patient voids at the conclusion of the study, the effective radiation dose from 70 to $200 \mathrm{MBq}$ of MAG3 or DTPA is approximately $10 \%-35 \%$ of the yearly background radiation dose of $3 \mathrm{mSv}(300 \mathrm{mrem}) .{ }^{37} \mathrm{~A}$ statement regarding radiation dose was considered a local option.

Cortical and Pelvic ROIs-If there is a dilated pelvis in a nonobstructed kidney or mild dehydration leading to pelvic retention, a whole-kidney ROI may result in a prolonged $T_{\max }$ even when the kidney is normal, whereas a cortical ROI may give a normal appearing renogram curve. Cortical ROI are not appropriate for measuring relative function and the panel considered the use of cortical ROIs to be a local option. Similarly, a ROI assigned over activity retained in the renal collecting system may provide a better index of washout than a ROI over the whole kidney, particularly in patients with impaired parenchymal function; however, $T_{1 / 2}$ values based on pelvic ROIs have not been widely reported and the panel also considered pelvic ROIs to be a local option.

Acquiring and Reporting into Structure-Reporting into structure organizes and may fundamentally alter the way the interpreting physician thinks about the case as he or she produces the report. ${ }^{38}$ For certain procedures, such as diuresis renography, reporting into structure has, as a precondition, acquiring into structure. The structure specifies what elements should be acquired to assure the quality of the study as well as to assure a report that maximizes the diagnostic utility of the procedure. Adopting the structured format and acquiring into structure provides a means for physicians to assure the quality of diuresis renography studies performed in their departments; reporting into structure guides the assembly of the essential elements required to reach an informed conclusion. Information acquired into a consistent format has the additional advantage of easy retrieval and can be broadly analyzed to support medical research and quality improvement. Finally, the structured format provides a means for outside observers to assess quality; the essential elements are either present or they are not present. ISCORN supports the use of structured reporting using standardized data elements and recommends that structured diuresis renography reports be implemented as part of the laboratory accreditation process.

Draft Normal Report-A draft normal report has been developed that incorporates the essential and recommended elements and provides a structure and options that can be adapted to the specific needs of individual centers. 


\section{Draft Normal Report}

\section{Indication}

The patient is a [age] year old [male/female] referred for [list referral reason].

\section{Clinical History}

There are no reports of a recent serum creatinine, urological procedures or renal imaging studies [the most recent available serum creatinine dated ( ) is ( )].

\section{Procedure}

The patient [did/did not] receive additional hydration [which consisted of]. The patient voided before the procedure, voiding time was recorded and the patient received an intravenous injection of $[\mathrm{mCi} / \mathrm{M} \mathrm{Bq}]$ of $[\mathrm{MAG} 3 / \mathrm{DTPA} / \mathrm{EC} / \mathrm{OIH}]$ resulting in a radiation dose approximately()\% of the $3 \mathrm{mSv}$ yearly background radiation (Describe imaging protocol, 1 or 2 stages, dose and timing of furosemide, if administered.) The [MAG3/DTPA/ $\mathrm{EC} / \mathrm{OIH}$ ] clearance was calculated by the use of a camera-based, single blood sample, multiple blood sample technique. Data were analyzed by the use of a dedicated computer. ROIs were assigned over each kidney and the renal cortex; a (specify) background region was assigned and the relative uptake was calculated at (specify time interval and method used for calculation). In addition to the $T_{\max }$ and $T_{1 / 2}$ using whole-kidney ROIs, the following quantitative parameters (specify any additional quantitative parameters generated from whole kidney, parenchymal or pelvic ROIs) were also calculated. The patient voided at the conclusion of the study; the voided volume was measured and time of voiding recorded to measure urine flow rate. Pre- and post-void bladder images were obtained to calculate residual urine volume and a postvoid image of the kidney was obtained to evaluate gravityassisted drainage.

\section{Findings}

The study is of good quality. The (MAG3/EC/DTPA/OIH) clearance is $\mathrm{mL} / \mathrm{min} / 1.73 \mathrm{~m}^{2}$ compared with a normal range of to $\mathrm{mL} / \mathrm{min} / 1.73 \mathrm{~m}^{2}$. The relative uptake of the right and left kidneys is $\%$ and _ $\%$, respectively. The kidneys have a normal configuration, comparable size and no abnormal areas of decreased tracer activity. There is prompt uptake of the tracer in both kidneys with rapid excretion after furosemide; there is no significant retention in either collecting system. The time to peak counts is $<5$ minutes for both kidneys; after the administration of furosemide, the $T_{1 / 2}$ of the left kidney is (_) minutes and (_) minutes for the right kidney. The voided volume was (_) $\mathrm{mL}$, the residual urine volume was (_) $\mathrm{mL}$, and the urine flow rate was $\left(\_\right) \mathrm{mL} / \mathrm{min}$.

\section{Impression}

1. The relative uptake is (_) \% in the right kidney is and (_) \% in the left kidney.

2. The(MAG3/EC/DTPA) clearance is $\left(\_\right) \mathrm{mL} / \mathrm{min} / 1.73 \mathrm{~m}^{2}$ with a normal range of(_to__ $) \mathrm{mL} / \mathrm{min} / 1.73 \mathrm{~m}^{2}$.

3. The residual volume is (_) $\mathrm{mL}$.

4. Neither kidney is obstructed. There is prompt uptake of the tracer in both kidneys and rapid drainage from both collecting systems following furosemide administration. 


\section{Uncited Reference}

This section consists of references that are included in the reference list but are not cited in the article text. Please either cite each of these references in the text or, alternatively, delete it from the reference list. If you do not provide further instruction for this reference, we will retain it in its current form and publish it as an "un-cited reference" with your article. ${ }^{23}$

\section{Acknowledgments}

This work was supported in part by the National Institute of Biomedical Imaging and Bioengineering and the National Institute of Diabetes and Digestive and Kidney Diseases (NIDDK), R01-EB008838. We would also like to thank Stephen Brown, MD, and John Petros, MD, for their comments and input in the initial stages of developing this document and to Helena Balon, MD for her thoughtful review of the manuscript.

\section{References}

1. Blaufox MD. A brief history of the Radionuclides in Nephrourology Group (ISCORN). Semin Nucl Med. 2008; 38:2-8.

2. Taylor NJ, Aurell M, et al. Consensus report on ACE inhibitor renography for detecting renovascular hypertension. J Nucl Med. 1996; 37:176-1882.

3. Blaufox MD, Aurell M, Bubeck B, et al. Report of the radionuclides in nephrourology committee on renal clearance. J Nucl Med. 1996; 37:1883-1890. [PubMed: 8917197]

4. O'Reilly P, Aurell M, Britton K, et al. Consensus on diuresis renography for investigating the dilated upper urinary tract. J Nucl Med. 1996; 37:1872-1876. [PubMed: 8917195]

5. Prigent A, Cosgriff P, Gates GF, et al. Consensus report on quality control of quantitative measurements of renal function obtained from the renogram: International Consensus Committee from the Scientific Committee of Radionuclides in Nephrourology. Semin Nucl Med. 1999; 29:146-159. [PubMed: 10321826]

6. Piepsz A, Cosgriff P, Gares GF, et al. Consensus on renal cortical scintigraphy in children with urinary tract infection. Semin Nucl Med. 1999; 29:160-174. [PubMed: 10321827]

7. Dubovsky EV, Russell CD, Bischof-Delaloye A, et al. Report of the Radionuclides in Nephrourology Committee for evaluation of transplanted kidney (review of techniques). Semin Nucl Med. 1999; 29:175-188. [PubMed: 10321828]

8. Durand E, Blaufox MD, Britton KE, et al. International Scientific Committee of Radionuclides in Nephrourology (ISCORN) consensus on renal transit time measurements. Semin Nucl Med. 2008; 38:82-102. [PubMed: 18096466]

9. IMV Medical Information Division. Nuclear Medicine Census Market Summary Report. Vol. 4. Des Plaines, IL: IMV Limited; 2003. p. 7-11.

10. Fletcher JW, Woolf SH, Royal HD. Consensus development for producing diagnostic procedure guidelines: SPECT brain perfusion imaging with exametazime. J Nucl Med. 1994; 35:2003-2010. [PubMed: 7989985]

11. Hendel RC, Wackers FJ, Berman DS, et al. American Society of Nuclear Cardiology consensus statement: Reporting of radionuclide myocardial perfusion imaging studies. J Nucl Cardiol. 2003; 10:705-708. [PubMed: 14668786]

12. Douglas PS, Hendel RC, Cummings JE, et al. ACCF/ACR/AHA/ASE/ASNC/HRS/NASCI/RSNA/ SAIP/SCAI/SCCT/SCMR 2008 Health Policy Statement on Structured Reporting in Cardiovascular Imaging. J Am Coll Cardiol. 2009; 53:76-90. [PubMed: 19118730]

13. Brater CD. Diuretic therapy. N Engl J Med. 1998; 39:387-395. [PubMed: 9691107]

14. Hunsche A, Press H, Taylor A. Increasing the dose of furosemide in patients with azotemia and suspected obstruction. Clin Nucl Med. 2004; 26:149-153. [PubMed: 15162982]

15. Thrall, JH.; Ziessman, HA. Nuclear Medicine: The Requisites. St Louis, MO: Mosby Year Book; 1995. p. 300

16. Hall FM. Language of the radiology report: Primer for residents and way ward radiologists. AJR Am J Roentgenol. 2000; 175:1239-1242. [PubMed: 11044014] 
17. Society for Fetal Urology and the SNM, Pediatric Nuclear Medicine Council. . The "well tempered" diuretic renogram: A standard method to examine the asymptomatic neonate with hydronephrosis or hydroureteronephrosis. J Nucl Med. 1992; 33:2047-2051. [PubMed: 1432172]

18. Gordon I, Colarinha P, Fettich J, et al. Guidelines for standard and Diuretic Renogram in children. Eur J Nucl Med. 2001; 28:31-36.

19. Shulkin, BL.; Mandell, GA.; Cooper, JA., et al. [Accessed January 7, 2010] Procedure guideline for diuretic renography in children 3.0. Available at: http://interactive.snm.org/docs/162.pdf

20. Adeyoju AA, Burke D, Atkinson C, et al. The choice of timing for diuresis renography: The $\mathrm{F}+0$ method. BJU Int. 2001; 88:1-5. [PubMed: 11446835]

21. Kuyvenhoven J, Piepsz M, Ham H. When could the administration of furosemide be avoided? Clin Nucl Med. 2003; 28:732-737. [PubMed: 12972994]

22. Liu Y, Ghesani NV, Skurnick JH, et al. The F+0 protocol for diuretic renography results in fewer interrupted studies due to voiding than the F-15 protocol. J Nucl Med. 2005; 46:317-320.

23. Taylor A, Garcia EV, Binongo JNG, et al. Diagnostic performance of an expert system for interpretation of 99mTc MAG3 scans in suspected renal obstruction. J Nucl Med. 2008; 49:216224. [PubMed: 18199609]

24. Taylor A, Thakore K, Folks R, et al. Background subtraction in Tc-99m-MAG3 renography. J Nucl Med. 1994; 35:2054-2055.

25. Chaiwatanarat T, Padhy HK, Bomanji BB, et al. Validation of renal output efficiency as an objective quantitative parameter in the evaluation of upper urinary tract obstruction. J Nucl Med. 1993; 34:845-848. [PubMed: 8478722]

26. Rossleigh MA, Leighton DM, Farnsworth RH. Diuresis renography. The need for an additional view after gravity-assisted drainage. Clin Nucl Med. 1993; 18:210-213. [PubMed: 8462211]

27. Gordon I, Mialdea-Fernandex RM, Peters AM. Pelviureteric junction obstruction. The value of a post-micturition view in ${ }^{99 m}$ Tc DTPA diuretic renography. Br J Urol. 1988; 61:409-412. [PubMed: 3293688]

28. Piepsz A, Tondeur M, Ham H. Nora: A simple and reliable parameter for estimating renal output with or without frusemide challenge. Nucl Med Commun. 2000; 21:317-323. [PubMed: 10845219]

29. Wong DC, Rossleigh MA, Farnsworth RH. Diuretic renography with the addition of quantitative gravity-assisted drainage in infants and children. J Nucl Med. 2000; 41:1030-1036. [PubMed: 10855630]

30. Esteves FP, Taylor A, Manatunga A, et al. 99mTc-MAG3 renography: Normal values for MAG3 clearance and curve parameters, excretory parameters, and residual urine volume. AJR Am J Roentgenol. 2006; 187:W610-W619. [PubMed: 17114514]

31. Binongo JNG, Taylor A, Manatunga A, et al. Use of classification and regression trees in diuresis Renography. Acad Radiol. 2007; 14:306-311. [PubMed: 17307663]

32. Strauss BS, Blaufox MD. Estimation of residual urine and urine flow rates without urethral catheterization. J Nucl Med. 1970; 11:81-84. [PubMed: 5410926]

33. Levey, AS.; Madaio, MP.; Perrone, RD. The Kidney. In: Brenner, BM.; Rector, FC., editors. Laboratory Assessment of Renal Disease: Clearance, Urinalysis and Renal Biopsy. WB Saunders Company, Harcourt Brace; Philadelphia, PA: Jovanovich, Inc; 1991. p. 919-968.

34. Halkar R, Taylor A, Manatunga A, et al. Monitoring renal function: a prospective study comparing camera-based technetium-99m mercaptoacetyltriglycine clearance and creatinine clearance. Urology. 2007; 69:426-430. [PubMed: 17382136]

35. Brown SCW, Upsdell SM, O'Reilly PH. The importance of renal function for the interpretation of diuresis renography. Br J Urol. 1992; 69:121-125. [PubMed: 1537020]

36. Stabin M, Taylor A, Eshima D, et al. Radiation dosimetry for technetium-99m-MAG3, technetium-99m-DTPA, and iodine-131-OIH based on human biodistribution studies. J Nucl Med. 1992; 33:33-40. [PubMed: 1530968]

37. Sistrom CL, Honeyman-Buck J. Free text versus structured format: Information transfer efficiency of radiology reports. AJR Am J Roentgenol. 2005; 185:804-812. [PubMed: 16120938] 